\title{
Ser profesional sanitario en el siglo XXI: algunos apuntes ${ }^{*}$
}

\section{Being a health professional in the 21 st century: some notes ${ }^{*}$}

\section{Ser profissional sanitário no século XXI: alguns apontes*}

\author{
Juan Ignacio Rico Becerra ${ }^{1}$, Salvador Cayuela Sánchez ${ }^{2}$ \\ ${ }^{1}$ Doctor en Antropología Social. Facultad de Ciencias Sociosanitarias de la Universidad de Murcia. \\ ${ }^{2}$ Doctor en Filosofía y Antropología Social. Facultad de Filosofía de la Universidad de Murcia. \\ ${ }^{*}$ ) Una versión inicial de este artículo fue pronunciada como ponencia invitada del Acto de Bienvenida a la Promoción de \\ Residentes 2015 del Área III de Salud de la Región de Murcia. \\ Cómo citar este artículo en edición digital: Rico Becerra1, J.I., Cayuela Sánchez, S. (2017). Ser profesional sanitario en el siglo \\ XXI: algunos apuntes. Cultura de los Cuidados (Edición digital), 21(48). \\ Recuperado de < http://dx.doi.org/10.14198/cuid.2017.48.14> \\ Correspondencia: Juan Ignacio Rico Becerra. Facultad de Ciencias Sociosanitarias, Campus de Lorca, Universidad de Murcia. Av. \\ de las Fuerzas Armadas s/n 30.800, Lorca (Murcia). \\ Correo electrónico: Juan.rico@um.es \\ Recibido: 14/12/2016; Aceptado: 22/03/2017
}

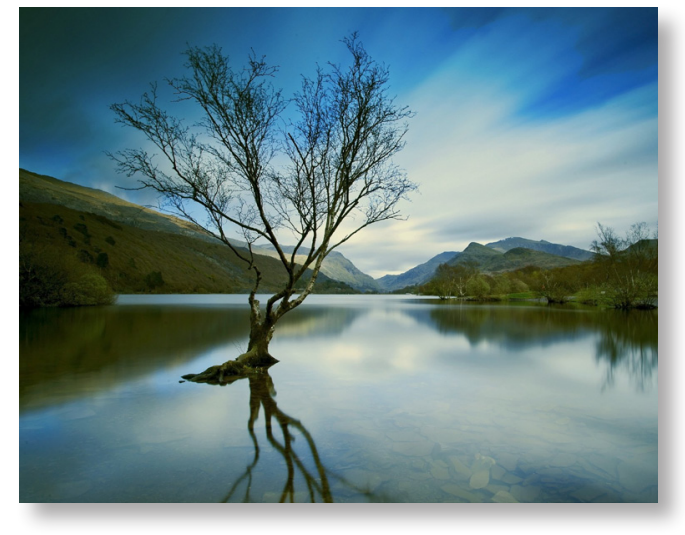

ABSTRACT

From 1970's theorists in History have noted the arrival of a new era known as Postmodernity, late-Modernity or reflexive Modernity. This new era, our current time, comes marked by the end of the 'great stories' that made western world comprehensible since ancient times, and cracking old certainties and putting the Subject in terms of social atomization and an accused self-responsibility. These ideological and social changes have brought profound consequences in many spheres (fields, scopes) in western human life, such as work, social (personal) relationships or politics, from where medicine and western practices don't (seem to) escape. On this basis, this article tries to analyze, firstly, the meanings and difficulties of this new era; from there, and secondly, it tries to understand the consequences that these changes and variations are assuming for professionals in the fields of medicine and health.

Keywords: change of era, medicine and western medical practice, epistemological Review, simple and reflexive scientific approach, new paradigm.

\section{RESUMEN}

Desde los años 70 del pasado siglo, los teóricos de la historia han señalado el advenimiento de una nueva época, adjetivada como postmodernidad, Modernidad tardía o Modernidad reflexiva. Este nuevo tiempo, el nuestro, vendría marcado por el fin de los grandes relatos que hicieron comprensible el mundo humano occidental desde la Antigüedad, resquebrajando las viejas certidumbres y arrojando al sujeto a la atomización social y la auto-responsabilidad exacerbada de su propia existencia. Estas transformaciones ideológicas y sociales han traído 
profundas consecuencias en diversas esferas de la vida humana en Occidente tales como el trabajo, las relaciones personales o la política, a las que no escapa, obviamente, la medicina y la praxis médica occidental. Partiendo de este hecho, el propósito de este artículo es intentar analizar, en primer lugar, las significaciones y problemáticas de ese cambio de época. y desde ahí, y en segundo lugar, tratar de comprender las consecuencias y variaciones que esas transformaciones están suponiendo para la medicina y sus profesionales sanitarios.

Palabras clave: cambio de época, medicina y praxis médica occidental, revisión epistemológica, cientificidad simple y reflexiva, nuevo paradigma.

\section{RESUMO}

Desde os anos 70 do passado século, os teóricos da história têm assinalado a chegada de uma nova época, adjetivada como postmodernidad, Modernidad tardia ou Modernidad reflexiva. Este novo tempo, o nosso, viria marcado pelo fim dos grandes relatos que fizeram compreensível o mundo humano ocidental desde a Antiguidade, resquebrajando as velhas certezas e arrojando ao sujeito à atomización social e a auto-responsabilidade exacerbada de sua própria existência. Estas transformações ideológicas e sociais têm trazido profundas consequências em diversas esferas da vida humana em Occidente tais como o trabalho, as relações pessoais ou a política, às que não escapa, obviamente, a medicina e a praxis médica ocidental. Partindo deste facto, o propósito deste artigo é tentar analisar, em primeiro lugar, as significações e problemáticas dessa mudança de época. e desde aí, e em segundo lugar, tratar de compreender as consequências e variações que essas transformações estão a supor para a medicina e seus profissionais sanitários.
Palavras-chave: Mudança de época, Medicina e praxis médica ocidental, Revisão epistemológica, Cientificidad simples e reflexiva, novo paradigma.

\section{INTRODUCCIÓN}

Como planteara Richard Sennett (2001) en su ya clásico estudio La corrosión del carácter, el trabajo ha dejado de ser en la actualidad para un gran número de personas una fuente de identidad personal, convirtiéndose en un agente erosionador del carácter. Trabajos precarios, flexibles, temporales, en los que resulta extremadamente complicado formarse e identificarse, precisamente, como profesional. Estas transformaciones del mundo laboral serían el resultado de toda una serie de procesos de diversa índole, que abarcarían desde lo económico y lo político, hasta lo cultural, lo tecnológico o lo ideológico. Ahora bien, ¿cómo están influyendo esas transformaciones del mundo laboral en los profesionales sanitarios del siglo XXI? (nos circunscribimos aquí a los profesionales sanitarios que desarrollan su labor dentro del ámbito de la sanidad pública, propia del llamado Estado del Bienestar europeo, ya que las precepciones, aspiraciones e identidades de los profesionales dedicados a la medicina liberal o privada, intuimos, deben ser ciertamente diferentes a los de nuestro ámbito de estudio y análisis), ¿debemos advertir dinámicas propias al sector, o sería exigible por el contrario tratar de entender la identidad, funciones $y$ perspectivas de estos especialistas en ese marco reflexivo e interrelacional tan propio de nuestra contemporaneidad?

\section{DESARROLLO DEL TEMA}

1. Para tratar de dar respuesta a estas cuestiones, es preciso atender en primer lugar a aque- 
llo a lo que hemos hecho ya referencia en el título, y que no puede ser obviado: a saber, lo que significa precisamente ese ser en el siglo XXI. Con esta expresión, en efecto, queremos referirnos a lo epocal (el vivir en el mundo de Ortega), y no tanto a lo ontológico (ese estar en el mundo que diría Heidegger). En este sentido, el tiempo que vivimos como contemporáneo viene marcado por el gran acontecimiento histórico de la Revolución Francesa (1789). Sería entonces el triunfo de la Ilustración y el nacimiento del hombre moderno, el brillante resultado de un proceso que encontraría en el Renacimiento su momento fundacional, y que habría ido desplegándose en acontecimientos tales como el descubrimiento de América por los occidentales (ese desvelamiento del Otro que marca, o al menos redefine, el inicio del Nosotros), el desarrollo de la imprenta, y el avance de una sociedad industrial y urbana que culminaría con la Revolución Industrial y el triunfo del capitalismo.

Ahora bien, el advenimiento de otros sucesos menos memorables ya en el siglo XX (las dos Guerras Mundiales, la invención de la bomba atómica, la constatación de los desastres del colonialismo, la crisis ecológica global, etc.), hicieron resquebrajarse esa triunfal concepción de la Modernidad y su inherente creencia en el progreso humano (Campillo, 1995). Entonces, y sobre todo a partir de los años 70 del siglo XX, es cuando parece abrirse un tiempo nuevo (que sería ya el actual), y que ha sido caracterizado como Posmodernidad (Jean-François Lyotard, Jean Baudrillard, Jaques Derrida, Gilles Deleuze y Gilles Lipovetsky en Francia; Gianni Vattimo en Italia; Peter Sloterdijk en Alemania; y Richard Rorty en EE.UU), Modernidad líquida (Zygmunt Bauman), Modernidad tardía (Anthony Giddens), Modernidad reflexiva o Sociedad del riesgo
(Ulrich Beck), incluso como El fin de la historia (Francis Fukuyama) o como Transmodernidad (Rosa María Rodríguez Mazda), por citar algunas de las etiquetas usadas más conocidas.

Al margen de las diferentes nomenclaturas y de sus matices de significación, todos estos autores hacen referencia, en efecto, a eso que a nosotros más nos interesa: a saber, los elementos que caracterizan nuestra epocalidad. En este punto, quizá haya sido el francés J. F. Lyotard (1984) quién más tempranamente supo comprender este tiempo nuevo, y algunas de sus características definitorias. Fue él precisamente el que nombró nuestra época como postmoderna en su exitoso ensayo $\mathrm{La}$ condición postmoderna de 1979, donde la define simple y llanamente como "la incredulidad hacia las metanarrativas”. En efecto, los cuatro grandes relatos de Occidente parecen haber sido finiquitados (Lyotard, 1986): el relato cristiano (Dios manda a morir a Jesucristo por la redención de los hombres prometiendo la salvación, un espacio de plenitud); el relato marxista (la burguesía liquida al feudalismo, y de ella surge el proletariado, que termina derrotándola e instaurando el Estado de la plenitud tras la revolución comunista); el relato del iluminismo (la narrativa de las luces y la razón, donde la Modernidad se desplegaría racionalmente acumulando verdad y poder, desterrándose para siempre la irracionalidad); $y$, finalmente, el relato capitalista (basado en la economía de mercado y su avance incontenible, lo que necesariamente debía redundar en la prosperidad para todos).

Lo común de estos grandes relatos, en efecto, es su compartida visión teleológica de la Historia, una concepción heredada del judeocristianismo que considera el tiempo histórico como irremediablemente conducente 
a un final redentor y de plenitud anunciada. El propio relato (o mejor, metarrelato) de la Modernidad sería también heredero de esta concepción progresista y lineal del tiempo humano, tan solo una interpretación metafísica más de la Historia. Ahora bien, ¿qué implica la muerte de estos grandes relatos, el fin de esta milenaria concepción teleológica de la Historia humana? La respuesta es casi lógica: la sola existencia de los pequeños relatos. En efecto, la postmodernidad es el tiempo de la exaltación del pequeño relato, de la fragmentación de la Historia, de la multiplicación de las justificaciones de la realidad, de la construcción siempre insuficiente de la completitud explicativa, de la renuncia a las pretensiones omniabarcantes de antaño. El hombre postmoderno, incrédulo de los metarrelatos, no dirige la totalidad de su vida conforme a un solo relato; su existencia se ha vuelto enormemente compleja, y no le queda ya más recurso que el microrrelato, capaz de dar sentido solo a una parte delimitada de la realidad y la existencia. En este nuevo tiempo, los microrrelatos solo responden al criterio fundamental de la utilidad, son esencialmente pragmáticos. Así, del diagnóstico de Nietzsche (Dios ha muerto) no se seguiría el nihilismo, sino la construcción de estos pequeños relatos que, aún siendo contradictorios, dotan de sentido a la existencia. El fin de la utopía, al tiempo, no impide la construcción de un espacio utópico en el seno de la intimidad, al modo de una utopía fragmentada para un mundo fragmentado. Como diría más tarde un gran maestro, Tierno Galván, nadie podrá cansarse de vivir si ha sido educado en el amor a lo finito.

2. Ahora bien, si los antiguos metarrelatos eran cosificadores (en su reducción de la subjetividad ante el aplastante devenir histórico de la plenitud), su extinción podría generar una progresiva liberación del hombre: emancipado de las grandes metas, el sujeto es capaz ya de soñar con la diversidad y la pluralidad. Así, la fragmentación, la babelización, no es considerada un mal, sino un estado positivo donde el hombre puede vivir libremente y gozar el presente siguiendo sus inclinaciones $y$ sus gustos (Vázquez Roca, 2011). A pesar de las consecuencias (la corrosión del carácter es quizá una de las más recalcitrantes), nada debe imponerse de modo imperativo y duradero. Y de ahí la condición eminentemente paradojal de la cultura postmoderna: materialista y psi, porno y discreta, renovadora y retro, consumista y ecologista, sofisticada y espontánea, espectacular y creativa, nihilista y escéptica, reivindicadora de lo plural y de lo particular. El compromiso con las minorías políticas, sexuales o lingüísticas, en un mundo globalizado, es quizá el mejor de los ejemplos (Castells, 1997): sin aparente contradicción, se puede ser a la vez cosmopolita y regionalista, o racionalista y eficaz en el trabajo, y discípulo intermitente de tal gurú oriental (Lipovetsky, 1989).

Es por ello que podemos afirmar que en la cultura postmoderna se da una segunda revolución individualista, un proceso de personalización (del "individualismo limitado" al “individualismo total”), que señala la nueva ética permisiva y hedonista: el culto al deseo desplaza el culto al esfuerzo. En efecto, el individualismo hedonista y personalizado se ha vuelto legítimo y ya no encuentra oposición, generando al tiempo un aislamiento suave, donde impera la búsqueda del ego y del propio interés. Aquella pérdida de coordenadas de la Modernidad (como ya apuntamos), no es ya tan positiva si entendemos cómo esa individualización es más anomia que autonomía: el individuo navega sin referencias estables, en 
un mundo en que la cosmovisión colectiva se desvanece dando paso a una multiplicidad de posibilidades que se deben dilucidar y negociar a cada paso. Lo que le impone la carga de elaborar su propia intimidad, asumiendo los riesgos que ello conlleva. Es por ello que este proceso de individualización exige identidades construidas "desde dentro" por el propio individuo, abandonados ya los patrones externos o colectivos de antaño. Identidades flexibles, en una sociedad en la que cada vez es menos probable mantener un mismo empleo, una misma familia, una misma posición social (Beck, 2003).

Ahora bien, ¿cuáles son las consecuencias sociales e individuales de este individualismo postmoderno? Fundamentalmente dos: por un lado, una privatización de las contradicciones; y por otro lado, la irrupción del narcisismo colectivo (Lipovetsky, 1989). En efecto, y en primer lugar, los individuos tienden a experimentar hoy los conflictos estructurales de la sociedad (pobreza, desempleo, formación, enfermedad, etc.), como asuntos que corresponde resolver en el ámbito de lo personal. Como intuyera ya a finales de los años ochenta Ulrich Beck (1998), los riesgos se han democratizado y generalizado en esta época de la Modernización reflexiva, convirtiéndose además en una cuestión principalmente personal: los riesgos se han individualizado. Se ha producido así una invisibilización de la dimensión social de los problemas, quedando todo circunscrito a la simple docilidad del individuo.

No obstante, para los teóricos anglofoucaultianos del grupo conocido como History of the Present Research Work (Nikolas Rose, Mitchel Dean, Thomas Osborne o Pat O’ Malley...), esto no supone una "negación de lo social", sino más bien una "metamorfosis en la conducción de la sociedad” (Vázquez García,

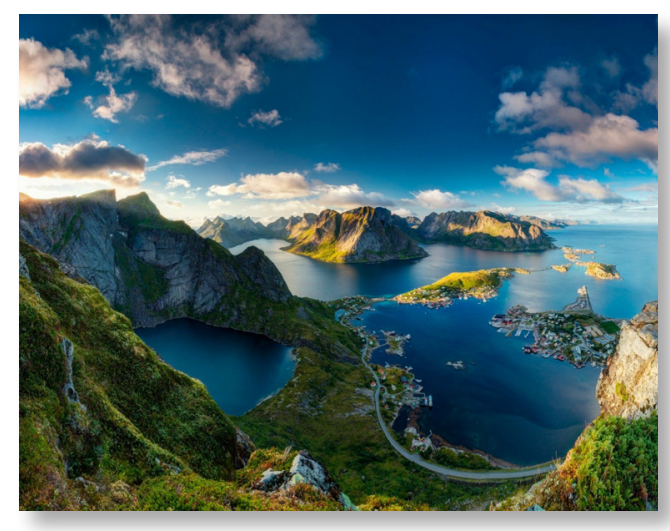

2005). Herederos de la concepción foucaultiana del gobierno como "conducción de conductas” (Foucault, 2004; Cayuela Sánchez, 2014), en esta nuestra Modernidad tardía cada uno debe hacerse responsable de su propia vida, gestionando de la mejor manera posible su capital estético, afectivo, psíquico, libidinal, etc., para encontrar así una vida auténtica. El gobierno neoliberal se ejercería por tanto de manera productiva sobre los individuos, no intentando reprimir una libertad primigenia, sino produciendo agentes y ámbitos de acción. Es por esto que las llamada "tecnologías psi" son tan importantes para la concepción social de este grupo: la psicología, la psiquiatría, la psicopedagogía, pero también el trabajo social, las asociaciones de anónimos, los preparadores físicos, etc., serían los dispositivos paradigmáticos de una gubernamentalidad neoliberal que busca llevar a sus últimas consecuencias las ideas de libertad y autocreación de sí mismo tan propias de nuestro tiempo (Rose, 1999; O'Malley, 1996).

$\mathrm{Y}$ como anunciamos, una segunda consecuencia de este individualismo postmoderno (conectada siempre con lo anterior) es el narcisismo colectivo, que impulsa el deseo de encontrarse en confianza, con seres que compartan las mismas preocupaciones inmediatas y circunscritas. Nos juntamos porque nos pare- 
cemos, porque estamos directamente sensibilizados por los mismos objetivos existenciales, porque nuestros intereses miniaturizados nos empujan a formar parte de colectivos identitarios: agrupaciones de viudos, de padres de hijos homosexuales, de alcohólicos, de tartamudos, de bulímicos, de madres lesbianas. El hombre postmoderno es, en efecto, un homo psycologicus que diría Lipovetsky (1989), diseñado por el gobierno neoliberal para buscar su propia realización personal, pero no por ello totalmente desconectado de lo social y replegado en su intimidad solipsista.

3. Estas consecuencias individuales y sociales que acarreó la ruptura de los metarrelatos no han sido las únicas. En efecto, a esta nueva multiplicidad de los discursos no escapa tampoco la razón y la ciencia, aquellos pilares sobre los que pretendía fundarse la Modernidad y el progreso humano. De hecho, podríamos afirmar que es la propia Modernidad y su transcurso problemático los que han propiciado su propio desarbolamiento, como la razón de la razón o la ciencia de la ciencia. Parafraseando a Hobbes y a su "homo homini lupus", la Modernidad es el lobo para la Modernidad, como para la razón y para la ciencia. Así, y como señalara Beck, la cientificación simple (tan propia de la Modernidad) ha dejado paso a una cientificación reflexiva (inherente a nuestra Modernidad tardía), donde no solo se somete a criterios científicos a la naturaleza, el hombre y la sociedad, sino también a la propia ciencia, sus productos, consecuencias y defecto (Beck, 1998). En esta nueva etapa se produce pues una desmonopolización del conocimiento, donde no es el fracaso de las ciencias, sino su propio y desmedido éxito lo que las ha destronado, provocando una relativización de sus originarias pretensiones de validez
(Rico Becerra, 2010). Como señala Sepúlveda Galeas (2011), retomando la expresión de Deleuze y Guattari (2002), diríamos que se activa un proceso de desterritorialización en ciertos ámbitos del saber, abriéndose espacios para la articulación de otros saberes que puedan maximizar las oportunidades de contradicción, o dicho de otro modo, abriéndose la posibilidad para la entrada en escena de saberes otros cuyo propósito sea la minimización de la dominación.

Así, mientras que la sociedad moderna creía en el futuro, en la ciencia y en la técnica, en la postmodernidad muere el optimismo tecnológico y científico. La interpretación en este punto es una condición necesaria para que podamos conocer la realidad, para que nos podamos relacionar con ella. No es extraño constatar por tanto que en nuestro tiempo postmoderno la Hermenéutica sea el gran objeto de la ciencia: lo importante no son los hechos, sino sus interpretaciones (Nietzsche, 1998; Gadamer, 1993). La certeza de un hecho no es más que eso, una verdad relativamente interpretada y por lo mismo, incierta.

Todo esto permite que lo apócrifo (ya sea Occidental u Oriental) conviva con lo oficial. En el caso de la medicina, la acupuntura, la naturopatía, la homeopatía, las psicoterapias (Biofeedback, Rebirthing, Bioenergía, Grito Primal, etc.), u otras "terapias suaves", conviven con nuestra medicina (también denominada, con más o menos acierto, convencional, occidental, cosmopolita, científica, biomedicina, alopatía, etnomedicina fisiológicamente orientada...). En este punto, muchas de estas terapias tradicionales/alternativas/complementarias enfatizan la subjetivación de la enfermedad, donde el enfermo no debe sufrir su estado de manera pasiva (ya no es paciente), sino que debe entenderse como responsable 
de su salud, de la asunción de riesgos, de sus sistemas de defensa... (es el llamado Nuevo Prudencialismo, diferente del veteroprudencialismo del siglo XIX y XX donde la sociedad a través del Estado Providencia tenía un papel capital) gracias a las potencialidades de la autonomía psíquica. Y aquí entra también, claro está, lo relativo al cuerpo: su cuerpo es usted, quiéralo, cuídelo, alardee de él. Es el momento de los más variados mensajes cosméticos: es preciso mantenerse siempre joven, se revaloriza el cuerpo anulando la tradicional concepción cristiana (la carne es débil, el origen de todos los males y del pecado), se populariza una gran variedad de dietas, irrumpen gimnasias (y gimnasios) de todo tipo, tratamientos revitalizantes y rejuvenecedores, potenciándose y normalizándose las cirugías estéticas.

4. Llegamos ya, se intuye, a la segunda parte de nuestro artículo avanzada en el título: ser profesional sanitario, en nuestro caso, obviamente, en el marco de la medicina occidental. En este punto, y en primer lugar, es preciso tener presente que somos profesionales sanitarios adscritos a un determinado sistema médico (entendido como el conjunto de concepciones y prácticas relacionadas, que se despliegan en los procesos de salud, enfermedad y atención), y dentro de un modelo estatal (el Estado del Bienestar, aunque debilitado) donde la sanidad es mayoritariamente pública y gratuita.

Como antes señalamos (y no podía ser de otro modo), el análisis crítico y reflexivo característico de nuestro tiempo (la revisión epistemológica) también pone la mirada en nuestra medicina. Y de esta mirada crítica que se hace al modelo médico occidental, creemos que puede ser de interés revisar dos aspectos por la repercusión que hoy tienen: en primer lugar, el que recoge el ya clásico estudio de Iván Illich (1975), Némesis médica, sobre los aspectos sociales y políticos de la medicalización (entendida esta como el proceso por el que ciertos fenómenos que formaban parte de otros campos, como la educación, la ley, la religión, etc., han sido redefinidos como fenómenos médicos -Márquez \& Meneu, 2003-). Así, Illich considera que la medicalización de la vida tiene efectos iatrogénicos que se pueden agrupar en tres categorías: por un lado, la iatrogenia clínica, donde los agentes patógenos son los medicamentos, los médicos o los hospitales. Por otro lado, la iatrogenia social, donde lo perjudicial no deriva de la acción técnica directa (como en la iatrogenia clínica), sino de la dependencia creciente de la intervención profesional, bajo el supuesto de que la medicina podrá proporcionarnos una salud cada vez mejor y satisfacer todos los deseos que las limitaciones de nuestro cuerpo nos impida realizar. Y finalmente, la iatrogenia estructural, por la que la medicina clínica convierte en técnicos (apropiándose de ellos) ciertos aspectos que la/s cultura/s tradicionalmente han integrado y dotado de sentido, tales como el dolor o la muerte; es de recibo destacar que nuestra medicina cada vez es más consciente de las anteriores demandas, hoy se habla, por ejemplo, de prevención cuaternaria (Krumholz, 2013; Gérvas, Gavilán Moral \& Jiménez de Gracia, 2012), es decir, la que debiera primar sobre cualquier otra opción preventiva, diagnóstica o terapéutica, algo así como la versión práctica del primum non nocere. Pero con todo, ¿es suficiente?, si se nos permite preguntarlo con otras palabras, ¿contentaríamos a Ivan Illich?

Un segundo aspecto de la medicina occidental señalado por esta postmoderna mirada reflexiva es su intrínseco carácter biológico y biologicista. La atención a esta cuestión comienza a revisarse de hecho en los años 70 
(década que recordemos vio nacer las nuevas concepciones sobre nuestra actualidad), e impulsada por autores como Horacio Fábrega (1974), Arthur Kleinman(1978) o Allan Young (1982). En este punto, es destacable la aportación de Kleinman, quién (a partir de las propuestas del antropólogo Clifford Geertz -1987-) pasa a considerar la medicina occidental como un sistema médico entre otros, existente dentro de un marco cultural específico, y que como tal puede ser analizado reflexivamente: entender la ideología que lo sustenta, cómo construye la enfermedad, sus jerarquías inherentes, las posiciones de rol/status, la socialización y el proceso de inserción de sus profesionales, etc.

De todo lo aportado en esta dirección, es destacable la contribución realizada sobre la triple dimensión de la enfermedad. Es aquí donde se imputa la excesiva mirada que la medicina occidental pone en lo biológico (ámbito denominado en inglés, Disease), dejando en un segundo plano tanto la dimensión subjetiva (Illness) como la social (Sickness). En efecto, la Disease (traducible como enfermedad o patología), designa la dimensión orgánica de la enfermedad, aquellas anomalías funcionales o estructurales de base orgánica que pueden ser objetivadas: esto es, observadas directamente a través de sus signos o por otros medios. La Illness (entendida como dolencia o padecimiento), hace referencia por su parte a la dimensión cultural de la enfermedad, y especialmente a la construcción semiótica, semiológica y fenomenológica de los síntomas y otras formas de expresión; al tiempo, designa el movimiento por el cual los procesos patológicos orgánicos o sensaciones de malestar son reconvertidos en experiencias individuales significativas. Finalmente, la Sickness alude a la dimensión social de la enfermedad, y por ende a las rela-

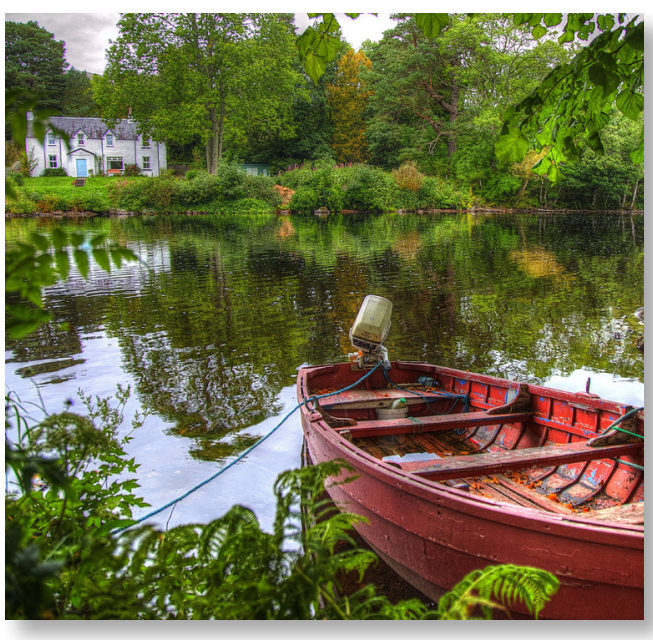

ciones sociales en donde se insertan los procesos de enfermedad, sus articulaciones ideológicas, socio-políticas y económicas (Comelles \& Martínez Hernáez, 1993); en este punto, el significado social de enfermedades como el SIDA o el cáncer pueden ser tremendamente esclarecedores.

Con todo, el hecho de que nuestra medicina centre su atención en la dimensión disease de la enfermedad, no significa, por lo demás, que no tenga presente la existencia de las otras dos. Esto es así, taxativamente, en la disciplina Enfermería, ya que siendo parte de la medicina convencional siempre ha puesto de manifiesto un interés especial por los aspectos socio-culturales, apostando por el enfoque holístico en la atención, al considerar a las personas en su completud (bio-psico-social) e insertas en la familia y la comunidad. Si bien, por desgracia, otra cosa es la relativa influencia que ha tenido en el modelo médico occidental, lo que se puede explicar por su tradicional subalternidad al mismo. En este sentido, creemos que Amezcua (2004) atina al señalar: el cambio, el poder y el conocimiento como los aliños que no deberían faltar en los planes de formación de Enfermería si se quiere estar preparados para los retos de la posmodernidad. 
5. Cerradas ya las dos líneas de análisis que han estructurado nuestro texto y guiado nuestras cuestiones, quizá estemos en condiciones de intentar comprender las problematizaciones que flanqueaban nuestro enunciado inicial: ser profesional sanitario en el siglo XXI. Indudablemente, este nuestro nuevo tiempo (que al margen de cómo lo denominemos trasciende sin duda a la Modernidad) genera múltiples retos para todos, incluidos por supuesto los profesionales sanitarios. Claro está, la necesidad del cambio que acompaña a la adaptación epocal no es nueva. Piénsese, por ejemplo, en aquellos profesionales de la salud que transitaron entre la Edad Media y el Renacimiento, o incluso entre la Alta y la Baja Edad Media (siglos X-XI), cuando nacen las primeras universidades (Escuela Salernitana), y se ponen en primera línea los textos de la medicina hipocrático-galénica, contrastando radicalmente con el anterior oscurantismo altomedieval. O si nos aproximamos temporalmente, lo que supuso para la medicina el nuevo enfoque de la teoría microbiana de Pasteur y Koch, frente a la teoría miasmática vinculada al higienismo.

Pero es que además, estos cambios internos en la medicina occidental tampoco pueden ser entendidos al margen de toda una serie de acontecimientos sociales, económicos, políticos y culturales que los propiciaron. Difícil entender la introducción de muchos textos y prácticas de la medicina antigua sin las traducciones realizadas en Toledo e impulsadas por el Rey Alfonso X, en pleno proceso de conquista a los musulmanes del sur de la Península Ibérica. Imposible también comprender el Renacimiento, con sus imágenes del mundo y del hombre (recuérdese el interés de Leonardo Da Vinci por las disecciones), sin atender a la caída en 1453 de Constantinopla, y la posterior llegada a Italia de un gran número de intelectuales, médicos, artistas, etc., herederos del gran Imperio Romano, y portadores de conocimientos y técnicas olvidados en Occidente. ¿Cómo comprender las percepciones, posiciones sociales e imaginarios de los profesionales de la medicina en Europa (y de los propios usuarios) durante la segunda mitad del siglo XX, sin atender a las circunstancias socioeconómicas y políticas tan propias de la Guerra Fría, y de la lucha occidental (tanto ideológica como militar) contra el comunismo soviético?

También en nuestra más radical actualidad, en esta época postmoderna donde las grandes ideologías y concepciones del mundo parecen haber dejado paso al hedonismo y al individualismo extremos, debemos tratar de comprender qué hemos de hacer para desempeñar nuestra labor como profesionales sanitarios. Sin duda alguna, las nuevas percepciones del cuidado de sí que portan hoy muchos de los usuarios de los servicios de salud, sus reivindicaciones de nuevas técnicas de sanación, sus inquietudes alimentarias..., son innegablemente síntomas de este tiempo nuevo en el que la exigencia de autorresponsabilidad (tan propia de la ideología neoliberal) amenaza con corromper (consecuencia quizá no meditada pero, seguro, alimentada por ciertos sectores políticos y empresariales) la percepción social de la medicina pública y de sus profesionales. Qué gran diferencia con ese trato reverencial con que el cliente recibía al profesional de la salud en aquel tiempo de práctica privada de la medicina de principios del siglo XX, y superviviente aún en tantos de nuestros mayores. En ambos casos, sin duda, memoria viva y encarnada, actitudes que obligan al profesional sanitario a comprender su tiempo, a entender las significaciones que definen la práctica médica, y lo posicionan en el espacio social. 


\section{CONCLUSIONES}

Para ir concluyendo, nos parece oportuno recordar lo que proponen Davis-Floyd y St. John (2004) a propósito de los modelos tecnocrático o tecnomédico, humanista o biopsico-social y/u holístico o energético por el que se mueven las distintas concepciones y prácticas de los distintos Sistemas Médicos (la medicina convencional lo haría fundamentalmente entre el primero y el segundo de esos modelos, ya que el ámbito de las especialidades médicas estaría más próximo a la tecnomedicina, mientras que la Atención Primaria, lo estaría del modelo humanista). Pues bien, desde este marco contextual sugerir que la Weltanschauung que al respecto dibuja la nueva epocalidad, trasciende incluso el segundo de los modelos, el humanista (que como sabemos apuesta por la conexión cuerpo-mente, considera el cuerpo como un organismo, equilibra la ciencia y la tecnología con el humanismo, propone el diagnóstico y curación de fuera hacia adentro y de adentro hacia fuera, apuesta por la toma de decisiones compartidas, está abierto a otras modalidades de sanación...), coqueteando, cada vez más, con parámetros que podemos inscribir de pleno en el modelo holístico, a saber, unidad cuerpo-mente-espíritu, el cuerpo como sistema de energía interconectado con otros sistemas energéticos, diagnóstico y curación de adentro hacia fuera, autoridad y responsabilidad como inherentes a cada individuo, la ciencia y la tecnología colocadas al servicio de cada individuo, abarcar múltiples modalidades de sanación... Que conste que con esto, no aseveramos que este último referente sea el definitivo o el "más" científico (esta cuestión no se dirime en este texto). Lo que sí quisiéramos subrayar es que la medicina Occidental, como modelo médico hegemónico (Menéndez \& Di
Pardo, 1994) y oficial en nuestro medio, no puede opacar una realidad como esta. Por lo que ya no se puede caer en el conocido como efecto tomate, es decir, ignorar o rechazar un tratamiento eficaz para una determinada enfermedad por no tener sentido con respecto a las teorías aceptadas sobre los mecanismos de las enfermedades y las acciones de las drogas (Goodwin \& Goodwin, 1984). La OMS (2002) ya advertía el enconamiento entre la medicina alopática y las medicinas tradicionales (ayurveda hindú, tradicional china, unani árabe...) y alternativas/complementarias, considerando el entusiasmo no crítico de las segundas frente al escepticismo no informado de la primera; a la vez que la propia Directora General, la Dra. Margaret Chan, animaba a su integración: "No tiene por qué haber conflicto entre la medicina tradicional y la medicina occidental. En el marco de la atención primaria, ambas pueden combinarse de forma armoniosa y beneficiosa, en un sistema que aproveche lo mejor de cada una y compense también las deficiencias de cada una. Ahora bien, esto no es algo que vaya a ocurrir espontáneamente: es preciso tomar deliberadamente decisiones normativas. Pero es posible hacerlo" (OMS, 2013).

Quizás Thomas Kuhn (1971), de todo esto y en relación a la influencia que los cambios epocales provocan en la ciencia normal (lo que por extensión afectaría a nuestra medicina), advertiría de un momento de crisis en la misma. Si este fuera nuestro caso: ¿cuánto durará este lapso?, ¿en qué grado afectará a la medicina oficial?, ¿será del suficiente calado para abrir las puertas de otro nuevo paradigma o mejor (si seguimos con precisión al filósofo de la ciencia de Cincinatti) de otra matriz disciplinaria, o simplemente integrará otros sistemas médicos (holísticos) bajo el paraguas de la medicina convencional?... 
Finalmente, apuntar, que lo presentado en estas páginas deviene de algunas reflexiones realizadas sobre el particular en nuestro medio, sabedores, de que como siempre, en las cuestiones derivadas de lo humano, se abren más preguntas que respuestas se dan. Lo que por otro lado debe servir de acicate para que las nuevas cosmovisiones que está generando la modernidad tardía sean tenidas muy en cuenta por nuestra medicina y sus profesionales sanitarios, que son quienes, en definitiva, han de actuar como tales en esta nueva epocalidad. La cuestión dilucidada, nada más y nada menos, es la de ser profesional de la salud en el siglo XXI. Es obvio que el crono ya está en marcha y no hay vuelta atrás, no perdamos, pues, de vista esta senda, considerando, como corresponde en el año 15 del siglo XXI, los múltiples elementos en juego.

\section{BIBLIOGRAFÍA}

- Amezcua Martínez, M. (2004). Cambio, poder y conocimiento, los aliños de la Enfermería en la posmodernidad. Cultura de los Cuidados, (15), 5-8.

- Beck, U., \& Beck-Gernsheim, E. (2003). La individualización. El individualismo institucionalizado y sus consecuencias sociales y políticas. Barcelona: Paidós.

- Beck, U. (1998). La sociedad del riesgo. Hacia una nueva Modernidad. Barcelona: Paidós.

- Campillo, A. (1995). Adiós al progreso. Una meditación sobre la historia. Barcelona: Anagrama.

- Castells, M. (1997). La era de la información: economía, sociedad y cultura. Vol. 1. La sociedad real. Madrid: Alianza.

- Cayuela Sánchez, S. (2014). Por la grandeza de la patria. La biopolítica en la España de Franco (1939-1975). Madrid: Fondo de Cultura Económica.

- Comelles, J.M., \& Martínez Hernáez, A. (1993). Enfermedad, Cultura y Sociedad. Madrid: Eudema.

- Davis-Floyd, R., \& St. John, G. (2004). Del Médico al Sanador. Buenos Aires: Creavida.
- Deleuze, G., \& Guattari F. (2002). Mil mesetas: Capitalismo y esquizofrenia. Barcelona: Editorial Pretextos.

- Fábrega, H. (1974). Disease and Social Behavior: An interdisciplinary Perspective. Cambridge: The Mit Press.

- Foucault, M. (2004). Sécurité, territoire, population. Cours au Collège de France 1977-1978. Paris: Gallimard/Seuil.

- Gadamer, H. G. (1993). Verdad y Método: Fundamentos de una Hermenéutica Filosófica. Salamanca: Sígueme.

- Geertz, C. (1987). La interpretación de las culturas. México DF: GEDISA.

- Gérvas, J., Gavilán Moral, E., \& Jiménez de Gracia, L. (2012). Prevención cuaternaria: es posible (y deseable) una asistencia sanitaria menos dañina. Actualización en Medicina de Familia, 8 (6): 312-317.

- Goodwin, J.S., \& Goodwin, J.M. (1984). The Tomato Effect: Rejection of Highly Efficacious Therapies. Journal of the American Medical Association, (251), 23887-23890.

- Illich, I. (1975). Némesis médica. La expropiación de la salud. Barcelona: Barral editores.

- Kleinman, A. (1978). What Kind of Models for the Anthropology of Medical Systems? American Anthropologist, (80), 661-665.

- Krumholz, H.M. (2013). Post-Hospital Syndrome: An Acquired, Transient Condition of Generalized Risk. The New England Journal of Medicine, 368, 100-102.

- Kuhn, T. (1971). La estructura de las revoluciones cientificas. México DF: Fondo de Cultura Económica.

- Lipovetsky, G. (1989). Lère du vide. Essais sur l'individualisme contemporain. Paris: Gallimard.

- Lyotard, J.F. (1984). La condición postmoderna. Informe sobre el saber. Madrid: Cátedra.

-Lyotard, J.F. (1986). La postmodernidad explicada a los niños. Barcelona: Gedisa.

- Márquez, S., \& Meneu, R. (2003). La medicalización de la vida y sus protagonistas. Gestión Clínica y Sanitaria, 5 (2): 47-53.

- Menéndez, E., \& Di Pardo, R. (1994). De algunos alcoholismos y algunos saberes. Atención Primaria $y$ proceso de alcoholización. México DF: CIESAS.

-Nietzsche, F. (1998). Sobre verdad y mentira en sentido extramoral. Madrid: Tecnos. 
- O’Malley, P. (1996). Risk and Responsability. En A. Barry, T. Osborne, \& N. Rose, Foucault and Political Reason. Liberalism, Neoliberalism and Rationalities of Power (págs. 283-299). Chicago: University of Chicago Press.

- Ortiz Lobo, A., \& Ibáñez Rojo, V. (2011). Iatrogenia y prevención cuaternaria en salud mental. Revista Española de Salud Pública, 85 (6), 513-523.

- Organización Mundial de la Salud (2002). Estrategias de la OMS sobre medicina tradicional 2002-2005. Ginebra: OMS.

- Organización Mundial de la Salud (2013). Estrategias de la OMS sobre medicina tradicional 2014-2023. Ginebra: OMS.

-Rico Becerra, J.I. (2010). Enfermería, Cuidado y Ciencia. Cultura de los Cuidados, (28), 76-82.

- Rose, N. (1999). Governing the Soul. The Shaping of the
Private Self. London: Free Books.

- Sennet, R. (2001). La corrosión del carácter. Las consecuencias personales del trabajo en el nuevo capitalismo. Barcelona: AnagraMA.

- Sepúlveda Galeas, M. (2011). El riesgo como dispositivo de gobierno: neoprudencialismo y subjetivación. Revista de Psicología de la Universidad de Chile, 20 (2): 103-124.

- Vázquez García, F. (2005). Tras la autoestima. Variaciones sobre el yo expresivo en la Modernidad tardía. San Sebastian: Gakoa.

- Vásquez Rocca, A. (2011). La Posmodernidad; nuevo régimen de verdad, violencia metafísica y fin de los Metarrelatos. Nómadas, (29), 285-300.

- Young, A. (1982). The Anthropologies off Illness and Sickness. Annual Review of Anthropology, (11): 257-285.

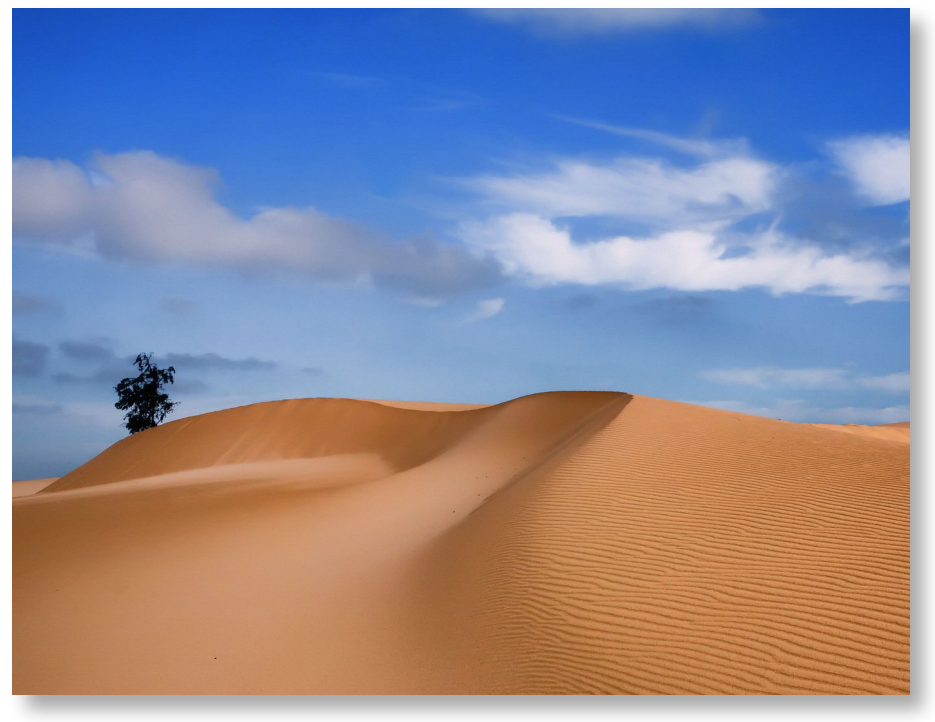

